\title{
A search for neutrino emission from the Fermi bubbles with the ANTARES telescope
}

\author{
The ANTARES Collaboration
}

S. Adrián-Martínez ${ }^{1}$, A. Albert ${ }^{2}$, I. Al Samarai ${ }^{3}$, M. André4 ${ }^{4}$, G. Anton ${ }^{6}$, S. Anvar ${ }^{7}$, M. Ardid ${ }^{1}$, T. Astraatmadja ${ }^{8, b}$, J.-J. Aubert ${ }^{3}$, B. Baret ${ }^{9}$, J. Barrios-Martí ${ }^{10}$, S. Basa ${ }^{11}$, V. Bertin ${ }^{3}$, S. Biagi ${ }^{12,13}$, C. Bigongiari ${ }^{10}$, C. Bogazzi $^{8}$, B. Bouhou ${ }^{9}$, M. C. Bouwhuis ${ }^{10}$, J. Brunner ${ }^{3}$, J. Busto ${ }^{3}$, A. Capone ${ }^{14,15}$, L. Caramete ${ }^{16}$, C. Cârloganu ${ }^{17}$, J. Carr ${ }^{3}$, S. Cecchini ${ }^{12}$, Z. Charif $^{3}$, Ph. Charvis ${ }^{18}$, T. Chiarusi ${ }^{12}$, M. Circella ${ }^{19}$, F. Classen ${ }^{6}$, R. Coniglione ${ }^{20}$, L. Core $^{3}$, H. Costantini ${ }^{3}$, P. Coyle ${ }^{3}$, A. Creusot ${ }^{9}$, C. Curtil ${ }^{3}$, G. De Bonis ${ }^{14,15}$, I. Dekeyser ${ }^{21,22}$, A. Deschamps ${ }^{18}$, C. Donzaud ${ }^{9,23}$, D. Dornic ${ }^{3}$, Q. Dorosti ${ }^{24}$, D. Drouhin ${ }^{2}$, A. Dumas ${ }^{17}$, T. Eberl ${ }^{6}$, U. Emanuele ${ }^{10}$, A. Enzenhöfer ${ }^{6}$, J.-P. Ernenwein ${ }^{3}$, S. Escoffier ${ }^{3}$, K. Fehn 6 , P. Fermani ${ }^{14,15}$, V. Flaminio ${ }^{25,26}$, F. Folger ${ }^{6}$, U. Fritsch ${ }^{6}$, L. A. Fusco ${ }^{12,13}$, S. Galatà9 ${ }^{9}$ P. Gay ${ }^{17}$, S. Geißelsöder ${ }^{6}$, K. Geyer ${ }^{6}$, G. Giacomelli ${ }^{12,13}$, V. Giordano ${ }^{33}$, A. Gleixner ${ }^{6}$, J. P. Gómez-González ${ }^{10}$, K. Graf ${ }^{6}$, G. Guillard ${ }^{17}$, H. van Haren ${ }^{27}$, A. J. Heijboer ${ }^{8}$, Y. Hello ${ }^{18}$, J. J. Hernández-Rey ${ }^{10}$, B. Herold ${ }^{6}$, J. HößI ${ }^{6}$, C. Hugon ${ }^{5}$, C. W. James ${ }^{6}$, M. de Jong ${ }^{8, b}$, M. Kadler ${ }^{28}$, O. Kalekin', A. Kappes ${ }^{6, c}$, U. Katz ${ }^{6}$, P. Kooijman ${ }^{8,29,30}$, A. Kouchner ${ }^{9}$, I. Kreykenbohm ${ }^{31}$, V. Kulikovskiy ${ }^{5,32}$, a , R. Lahmann 6 , E. Lambard ${ }^{3}$, G. Lambard ${ }^{10}$, G. Larosa1 ${ }^{1}$, D. Lattuada ${ }^{20}$, D. Lefèvre ${ }^{21,22}$, E. Leonora ${ }^{33,34}$, D. Lo Presti ${ }^{33,34}$, H. Loehner ${ }^{24}$, S. Loucatos ${ }^{7,9}$, F. Louis ${ }^{7}$, S. Mangano ${ }^{10}$, M. Marcelin'11, A. Margiotta ${ }^{12,13}$, J. A. Martínez-Mora1 , S. Martini' ${ }^{21,22}$, T. Michael ${ }^{8}$, T. Montaruli ${ }^{21,35}$, M. Morganti ${ }^{25, d}$, C. Müller ${ }^{31}$, M. Neff ${ }^{6}$, E. Nezri ${ }^{11}$, D. Palioselitis ${ }^{8, e}$, G. E. Păvălaş ${ }^{16}$, C. Perrina ${ }^{14,15}$, V. Popa ${ }^{16}$,

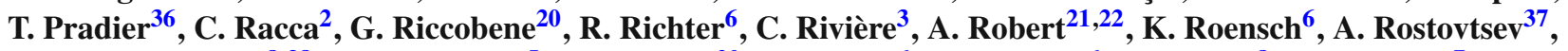
D. F. E. Samtleben ${ }^{8,38}$, M. Sanguineti ${ }^{5}$, P. Sapienza ${ }^{20}$, J. Schmid ${ }^{6}$, J. Schnabel ${ }^{6}$, S. Schulte ${ }^{8}$, F. Schüssler ${ }^{7}$, T. Seitz ${ }^{6}$, R. Shanidze ${ }^{6}$, C. Sieger ${ }^{6}$, F. Simeone ${ }^{14,15}$, A. Spies ${ }^{6}$, M. Spurio ${ }^{12,13}$, J. J. M. Steijger ${ }^{8}$, Th. Stolarczyk ${ }^{7}$, A. Sánchez-Losa ${ }^{10}$, M. Taiuti ${ }^{4,39}$, C. Tamburini' ${ }^{21,22}$, Y. Tayalati ${ }^{40}$, A. Trovato ${ }^{20}$, B. Vallage ${ }^{7}$, C. Vallée ${ }^{3}$, V. Van Elewyck ${ }^{9}$, M. Vecchi ${ }^{3, f}$, P. Vernin ${ }^{7}$, E. Visser ${ }^{8}$, S. Wagner ${ }^{6}$, J. Wilms ${ }^{31}$, E. de Wolf ${ }^{8,30}$, K. Yatkin ${ }^{3}$, H. Yepes ${ }^{10}$, J. D. Zornoza ${ }^{10}$, J. Zúñiga ${ }^{10}$

\footnotetext{
${ }^{1}$ Institut d'Investigació per a la Gestió Integrada de les Zones Costaneres (IGIC), Universitat Politècnica de València, C/Paranimf 1, 46730 Gandia, Spain

${ }^{2}$ GRPHE, Institut universitaire de technologie de Colmar, 34 rue du Grillenbreit, BP 50568, 68008 Colmar, France

${ }^{3}$ CPPM, Aix-Marseille Université, CNRS/IN2P3, Marseille, France

${ }^{4}$ Laboratory of Applied Bioacoustics, Technical University of Catalonia, Rambla Exposició, 08800 Vilanova i la Geltrú, Barcelona, Spain

5 INFN, Sezione di Genova, Via Dodecaneso 33, 16146 Genoa, Italy

${ }^{6}$ Erlangen Centre for Astroparticle Physics, Friedrich-Alexander-Universität Erlangen-Nürnberg, Erwin-Rommel-Str. 1, 91058 Erlangen, Germany

${ }^{7}$ Direction des Sciences de la Matière, Institut de recherche sur les lois fondamentales de l'Univers, Service d'Electronique des Détecteurs et d'Informatique, CEA Saclay, 91191 Gif-sur-Yvette Cedex, France

${ }^{8}$ Nikhef, Science Park, Amsterdam, The Netherlands

9 APC, Université Paris Diderot, CNRS/IN2P3, CEA/IRFU, Observatoire de Paris, Sorbonne Paris Cité, 75205 Paris, France

10 IFIC, Instituto de Física Corpuscular, Edificios Investigación de Paterna, CSIC, Universitat de València, Apdo. de Correos 22085, 46071 Valencia, Spain

${ }^{11}$ LAM, Laboratoire d'Astrophysique de Marseille, Pôle de l'Étoile Site de Château-Gombert, rue Frédéric Joliot-Curie 38, 13388 Marseille Cedex 13, France

12 INFN, Sezione di Bologna, Viale Berti-Pichat 6/2, 40127 Bologna, Italy

13 Dipartimento di Fisica dell’Università, Viale Berti Pichat 6/2, 40127 Bologna, Italy

14 INFN, Sezione di Roma, P.le Aldo Moro 2, 00185 Rome, Italy

15 Dipartimento di Fisica dell’Università La Sapienza, P.le Aldo Moro 2, 00185 Rome, Italy

16 Institute for Space Sciences, 77125 Bucharest, Măgurele, Romania

${ }^{17}$ Laboratoire de Physique Corpusculaire, Clermont Université, Université Blaise Pascal, CNRS/IN2P3, BP 10448, 63000 Clermont-Ferrand, France

${ }^{18}$ Géoazur, Université Nice Sophia-Antipolis, CNRS/INSU, IRD, Observatoire de la Côte d'Azur, Sophia Antipolis, France

${ }^{19}$ INFN, Sezione di Bari, Via E. Orabona 4, 70126 Bari, Italy

${ }^{20}$ INFN, Laboratori Nazionali del Sud (LNS), Via S. Sofia 62, 95123 Catania, Italy

${ }^{21}$ Mediterranean Institute of Oceanography (MIO), Aix-Marseille University, 13288 Marseille Cedex 9, France

22 Universit du Sud Toulon-Var, CNRS-INSU/IRD UM 110, 83957 La Garde Cedex, France
} 
${ }^{23}$ Université Paris-Sud, 91405 Orsay Cedex, France

${ }^{24}$ Kernfysisch Versneller Instituut (KVI), University of Groningen, Zernikelaan 25, 9747 AA Groningen, The Netherlands

25 INFN, Sezione di Pisa, Largo B. Pontecorvo 3, 56127 Pisa, Italy

${ }^{26}$ Dipartimento di Fisica dell'Università, Largo B. Pontecorvo 3, 56127 Pisa, Italy

${ }^{27}$ Royal Netherlands Institute for Sea Research (NIOZ), Landsdiep 4, 1797 SZ 't Horntje (Texel), The Netherlands

${ }^{28}$ Institut für Theoretische Physik und Astrophysik, Universität Würzburg, Am Hubland, 97074 Würzburg, Germany

${ }^{29}$ Universiteit Utrecht, Faculteit Betawetenschappen, Princetonplein 5, 3584 CC Utrecht, The Netherlands

${ }^{30}$ Instituut voor Hoge-Energie Fysica, Universiteit van Amsterdam, Science Park 105, 1098 XG Amsterdam, The Netherlands

${ }^{31}$ Dr. Remeis-Sternwarte and ECAP, Universität Erlangen-Nürnberg, Sternwartstr. 7, 96049 Bamberg, Germany

${ }^{32}$ Skobeltsyn Institute of Nuclear Physics, Moscow State University, Leninskie Gory, 119991 Moscow, Russia

${ }^{33}$ INFN, Sezione di Catania, Viale Andrea Doria 6, 95125 Catania, Italy

${ }^{34}$ Dipartimento di Fisica ed Astronomia dell'Università, Viale Andrea Doria 6, 95125 Catania, Italy

35 Département de Physique Nucléaire et Corpusculaire, Université de Genève, 1211 Geneva, Switzerland

${ }^{36}$ IPHC-Institut Pluridisciplinaire Hubert Curien, Université de Strasbourg et CNRS/IN2P3, 23 rue du Loess, BP 28, 67037 Strasbourg Cedex 2, France

${ }^{37}$ ITEP, Institute for Theoretical and Experimental Physics, B. Cheremushkinskaya 25, 117218 Moscow, Russia

${ }^{38}$ Universiteit Leiden, Leids Instituut voor Onderzoek in Natuurkunde, 2333 CA Leiden, The Netherlands

${ }^{39}$ Dipartimento di Fisica dell'Università, Via Dodecaneso 33, 16146 Genoa, Italy

${ }^{40}$ Laboratory of Physics of Matter and Radiations, University Mohammed I, B.P.717, 6000 Oujda, Morocco

Received: 23 August 2013 / Accepted: 2 December 2013 / Published online: 6 February 2014

(C) The Author(s) 2014. This article is published with open access at Springerlink.com

\begin{abstract}
Analysis of the Fermi-LAT data has revealed two extended structures above and below the Galactic Centre emitting gamma rays with a hard spectrum, the so-called Fermi bubbles. Hadronic models attempting to explain the origin of the Fermi bubbles predict the emission of highenergy neutrinos and gamma rays with similar fluxes. The ANTARES detector, a neutrino telescope located in the Mediterranean Sea, has a good visibility to the Fermi bubble regions. Using data collected from 2008 to 2011 no statistically significant excess of events is observed and therefore upper limits on the neutrino flux in $\mathrm{TeV}$ range from the Fermi bubbles are derived for various assumed energy cutoffs of the source.
\end{abstract}

\section{Introduction}

Analysis of data collected by the Fermi-LAT experiment has revealed two large circular structures near the Galactic Centre, above and below the galactic plane-the so-called Fermi bubbles [1]. The approximate edges of the Fermi bubble regions are shown in Fig. 1. These structures are characterised

a e-mail: vladimir.kulikovskiy@ge.infn.it

b Also at University of Leiden, Leiden, The Netherlands

${ }^{c}$ On leave of absence at the Humboldt-Universität zu Berlin, Berlin, Germany

d Also at Accademia Navale de Livorno, Leghorn, Italy

e Now at the Max Planck Institute for Physics, Munich, Germany

${ }^{\mathrm{f}}$ Now at Academia Sinica, 128 Academia Road, Section 2, Nankang, Taipei 115, Taiwan, ROC and National Central University, No.300, Jhongda Rd., Jhongli, Taoyuan 32001, Taiwan, ROC by gamma-ray emission with a hard $E^{-2}$ spectrum and a constant intensity over the whole emission region.

Signals from roughly the Fermi bubble regions were also observed in the microwave band by WMAP [2] and, recently, in the radio-wave band [3]. Moreover, the edges correlate with the X-ray emission measured by ROSAT [4]. Several proposed models explaining the emission include hadronic mechanisms, in which gamma rays together with neutrinos are produced by the collisions of cosmic-ray protons with interstellar matter [5-7]. Others which include leptonic mechanisms or dark matter decay would produce lower neutrino emission or none at all [1,6,8-10]. The observation of a neutrino signal from the Fermi bubble regions would play a unique role in discriminating between models.

The properties of the hypothesised neutrino emission are described in Sect. 2. An overview of the ANTARES neutrino detector is given in Sect. 3 and the neutrino event reconstruction is described in Sect. 4. The search for neutrino emission is performed by comparing the number of events in the Fermi bubble regions to the number found in similar off-zone regions (Sect. 5). The event selection optimisation is based on a simulation of the expected signal as described in Sect. 6 . The selected events are presented in Sect. 7 together with the significance and the upper limit on the neutrino flux from the Fermi bubbles.

\section{Estimation of the neutrino flux}

The estimated photon flux in the energy range $1-100 \mathrm{GeV}$ covered by the Fermi-LAT detector from the Fermi bubble regions is [1]: 


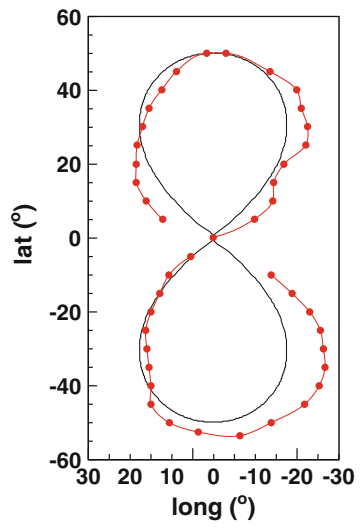

Fig. 1 Approximate edges (red line, circles) of the north and south Fermi bubbles respectively in galactic coordinates identified from the 1-5 GeV maps built from the Fermi-LAT data [1]. The contour line is discontinuous at the region of the Galactic Centre as the maps are severely compromised by the poor subtraction and interpolation over a large number of point sources in this region. The simplified shape of the Fermi bubbles used in this analysis (black line) has an angular area of $0.66 \mathrm{sr}$

$E^{2} \frac{\mathrm{d} \Phi_{\gamma}}{\mathrm{d} E} \approx 3-6 \times 10^{-7} \mathrm{GeV} \mathrm{cm}^{-2} \mathrm{~s}^{-1} \mathrm{sr}^{-1}$

Assuming a hadronic model in which the gamma-ray and neutrino fluxes arise from the decay of neutral and charged pions respectively, the $v_{\mu}$ and $\bar{v}_{\mu}$ fluxes are proportional to the gamma-ray flux with proportionality coefficients of 0.211 and 0.195 respectively [11]. With this assumption and using (1) the expected neutrino flux is:

$E^{2} \frac{\mathrm{d} \Phi_{v_{\mu}+\bar{v}_{\mu}}}{\mathrm{d} E}=A_{\text {theory }}$

$A_{\text {theory }} \approx 1.2-2.4 \times 10^{-7} \mathrm{GeV} \mathrm{cm}^{-2} \mathrm{~s}^{-1} \mathrm{sr}^{-1}$.

The neutrino flux, as well as the gamma-ray flux, is expected to have an exponential energy cutoff, so the extrapolation of (2) towards higher energies can be represented by:

$E^{2} \frac{\mathrm{d} \Phi_{v_{\mu}+\bar{v}_{\mu}}}{\mathrm{d} E}=A_{\text {theory }} \mathrm{e}^{-E / E_{v}^{\text {cutoff }}}$

The cutoff is determined by the primary protons which have a suggested cutoff $E_{p}^{\text {cutoff }}$ in the range from 1 to $10 \mathrm{PeV}$ [5]. The corresponding neutrino-energy cutoff may be estimated by assuming that the energy transferred from $p$ to $v$ derives from the fraction of energy going into charged pions ( $20 \%)$ which is then distributed over four leptons in the pion decay. Thus:

$E_{v}^{\text {cutoff }} \approx E_{p}^{\text {cutoff }} / 20$

which gives a range from 50 to $500 \mathrm{TeV}$ for $E_{v}^{\text {cutoff }}$.

\section{The ANTARES neutrino telescope}

The ANTARES telescope is a deep-sea Cherenkov detector which is located $40 \mathrm{~km}$ from Toulon (France), at a latitude of $42^{\circ} 48^{\prime} \mathrm{N}$ and at a mooring depth of $2,475 \mathrm{~m}$. The energy and direction of incident neutrinos are measured by detecting the Cherenkov light produced in water from muons originating in the charged-current interactions of $v_{\mu}$ and $\bar{v}_{\mu}$. The light is detected with a three-dimensional array of twelve detection lines comprising 885 optical modules, each containing a 10 inch PMT. More details on the detector construction, its positioning system and the time calibration can be found in [12-14].

The ANTARES detector started data-taking with the first five lines installed in 2007. The construction of the detector was completed, with installation of the last two lines, in May 2008. The apparatus has been operating continuously ever since. Its main goal is the detection of neutrinos produced by the cosmic sources. Muons and neutrinos created in cosmic-ray induced atmospheric showers provide the two main background components for the search for cosmic neutrinos. Although the more than $2 \mathrm{~km}$ of water above the detector acts as a partial shield against the atmospheric muons, the downgoing atmospheric muon background at these depths is still bigger than the expected signal. Therefore, the search for cosmic signal concentrates on upgoing events which corresponds to neutrinos which have crossed the Earth. Also, the optical modules are oriented downwards at $45^{\circ}$ to favour the detection of upgoing particles. The ANTARES neutrino telescope has an excellent visibility by means of the upgoing neutrinos to the Galactic Centre region and to the Fermi bubbles. Since atmospheric neutrinos may traverse the Earth and lead to upgoing tracks in the detector, any signal from the Fermi bubbles would be inferred by observing a significant statistical excess over the background. The signal-to-noise ratio can be improved by rejecting low-energy neutrino events, as the spectrum of the atmospheric neutrinos is steeper than the expected source spectrum.

\section{Track and energy reconstruction}

The track of a muon passing through the detector is reconstructed using the arrival time of the photons together with the positions and orientations of the photomultipliers. Details of the tracking algorithm are given in [15]. Only events reconstructed as upgoing have been selected. In addition, cuts on the reconstruction quality parameters have been applied in order to reject downgoing atmospheric muon events that are incorrectly reconstructed as upgoing tracks. These parameters are the quality $\Lambda$ of the track fit, which is derived from the track fit likelihood, and the uncertainty $\beta$ of the reconstructed track direction. The choice of the cut on $\Lambda$ fixes the 
amount of background from misreconstructed atmospheric muons in the neutrino sample. Neutrino simulations for an $E^{-2}$ neutrino spectrum have yielded a median angular resolution on the neutrino direction of less than $0.6^{\circ}$ for events with $\Lambda>-5.2$ and $\beta<1^{\circ}$ [15].

Shower-like events are identified by using a second tracking algorithm with $\chi^{2}$-like fit, assuming the hypothesis of a relativistic muon $\left(\chi_{\text {track }}^{2}\right)$ and that of a shower-like event $\left(\chi_{\text {point }}^{2}\right)$ [16]. Events with better point-like fit $\left(\chi_{\text {point }}^{2}<\chi_{\text {track }}^{2}\right)$ have been excluded from the analysis.

In this analysis the energy of the muons entered or born in the detector was estimated using Artificial Neural Networks, which are produced using a machine learning algorithm which derives the dependence between a set of observables and the energy estimate in a semi-parametric way [17]. The parameters used include the number of detected photons, and the total deposited charge. The median resolution for $\log _{10} E_{\operatorname{Rec}}$ is about 0.3 for muons with an energy of $10 \mathrm{TeV}$. The reconstructed energy $E_{\text {Rec }}$ is used to reject the atmospheric neutrino background while $\Lambda$ is used mostly to reject atmospheric muons. The choice of cuts on $\Lambda$ and $E_{\mathrm{Rec}}$ in this work is discussed in Sect. 6.

\section{Off-zones for background estimation}

A signal from the combined Fermi bubble regions is searched for by comparing the number of selected events from the area of both bubbles (on-zone) to that of similar regions with no expected signal (off-zones). The simplified shape of each Fermi bubble as used in this analysis is shown in Fig. 1.

Off-zones are defined as fixed regions in equatorial coordinates which have identical size and shape as the on-zone but have no overlap with it. In local coordinates, such off-zones have the same, sidereal-day periodicity as the on-zone and span the same fraction of the sky, but with some fixed delay in time. The size of the Fermi bubbles allows at maximum three non-overlapping off-zones to be selected. The on-zone and three off-zones are shown in Fig. 2 together with the sky visibility. The visibility of each point on the sky is the fraction of the sidereal day during which it is below the horizon at the ANTARES site (in order to produce upgoing events in the detector). The average visibility of the Fermi bubbles is 0.68 ( 0.57 for the northern bubble and 0.80 for the southern bubble) and it is the same for the off-zones.

Slightly changing detector efficiency with time and gaps in the data acquisition can produce differences in the number of background events between the on-zone and the three off-zones. In order to test for such an effect, firstly, the number of events in the off-zones is extracted from the data for various cuts ( $\left.\Lambda^{\text {cut }}, E_{\mathrm{Rec}}^{\mathrm{cut}}\right)$ and the difference in the event numbers between each pair of off-zones is calculated. This difference is compared with the statistical uncertainty and no

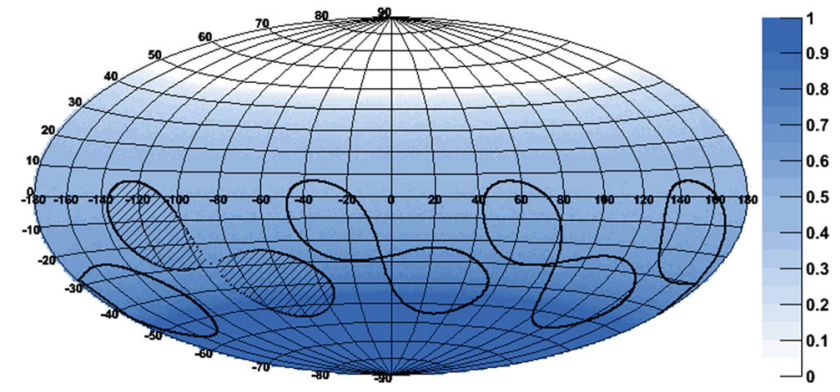

Fig. 2 Hammer equal-area map projection in equatorial coordinates $(\alpha, \delta)$ showing the Fermi bubble regions (on-zone) shaded area in the centre. The regions corresponding to the three off-zones are also depicted. The colour fill represents the visibility of the sky at the ANTARES site. The maximum on the colour scale corresponds to a 24 h per day visibility

excess is seen beyond the expected statistical fluctuations. Secondly, the number of events in the on-zone together with the average number of events in the three off-zones is tested using the simulated atmospheric background and the difference is found to be within the expectation from the statistical uncertainty. It can be concluded, therefore, that this effect is negligible.

\section{Event selection criteria}

The analysis adopts a blind strategy in which the cut optimisation is performed using simulated data for the signal and the background. The main quantities used to discriminate between the cosmic neutrino candidate events and the background from misreconstructed atmospheric muons and from atmospheric neutrinos are the tracking quality parameter $\Lambda$ and the reconstructed muon energy $E_{\text {Rec }}$.

The simulation chain for ANTARES is described in [18]. For the expected signal from the Fermi bubbles, the $v_{\mu}$ and $\bar{v}_{\mu}$ fluxes according to Sect. 2 are assumed, using four different cutoffs $E_{v}^{\text {cutoff }}:$ no cutoff $\left(E_{v}^{\text {cutoff }}=\infty\right), 500,100$ and $50 \mathrm{TeV}$. Atmospheric neutrinos are simulated using the model from the Bartol group [19] which does not include the decay of charmed particles.

Data in the period from May 2008, when the detector started to operate in its complete configuration, until December 2011 are used. The total livetime selected for this analysis amounts to 806 days. Figure 3 shows the distribution of data and simulated events as a function of the parameter $\Lambda$ for events arriving from the three off-zones. Here the events with at least ten detected photons and the angular error estimate $\beta<1^{\circ}$ are selected. The requirement on the number of photons removes most of the low-energy background events. The angular error condition is necessary in order to ensure a high angular resolution to avoid events originating from an off-zone region being associated with the signal region and vice versa. 


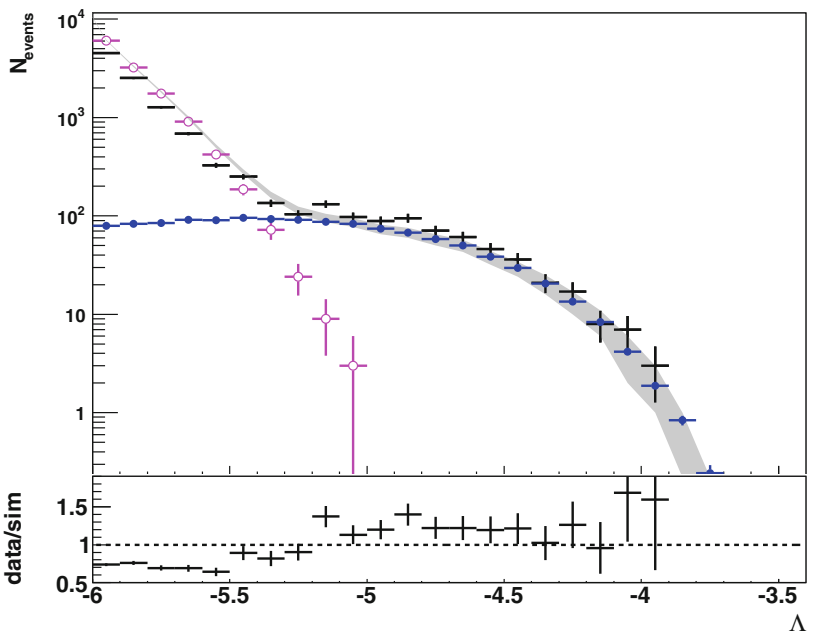

Fig. 3 Distribution of the fit-quality parameter $\Lambda$ for the upgoing events arriving from the three off-zones: data (black crosses), $68 \%$ confidence area given by the total background simulation (grey area), $\nu_{\mathrm{atm}}^{\mathrm{sim}}$ (blue filled circles), $\mu_{\mathrm{atm}}^{\mathrm{sim}}$ (pink empty circles); bin-ratio of the data to the total background simulation (bottom)

At $\Lambda \sim-5.3$ the main background component changes from the misreconstructed atmospheric muons to the upgoing atmospheric neutrino events as seen in Fig. 3. The flux of atmospheric neutrinos in the simulation is $23 \%$ lower than observed in the data. This is well within the systematic uncertainty on the atmospheric neutrino flux and the atmospheric flux from the simulations was scaled accordingly in the following analysis.

A comparison of the energy estimator for data and for atmospheric neutrino simulation is shown in Fig. 4 for the same event selection but with a stricter cut $\Lambda>-5.1$ to remove most of the misreconstructed atmospheric muons. The reconstructed energy of all simulated events has been shifted, $\log _{10} E_{\mathrm{Rec}}=\log _{10} E_{\mathrm{Rec}}^{\text {original }}+0.1$, in order to improve the agreement between data and simulations. This is within the estimated uncertainty of the optical module efficiency and the water absorption length [20, Figure 4.24].

The final event selection is optimised by minimising the average upper limit on the flux:

$\bar{\Phi}_{90 \%}=\Phi_{v_{\mu}+\bar{v}_{\mu}} \frac{\bar{s}_{90 \%}(b)}{s}$,

where $s$ is the number of events simulated with the flux $\Phi_{v_{\mu}+\bar{v}_{\mu}}$ from (4). The method uses an approach following Feldman and Cousins [21] to calculate signal upper limits with $90 \%$ confidence level, $\bar{s}_{90 \%}(b)$, for a known number of simulated background events $b$. This best average upper limit in the case of no discovery represents the sensitivity of the detector to the Fermi bubbles' flux [22]. Using (4) the average upper limit on the flux coefficient $A$ can be defined as:

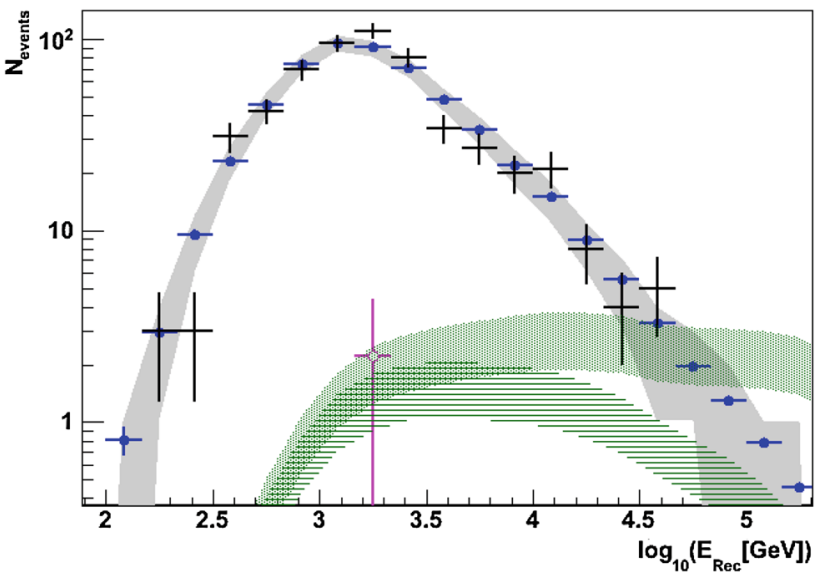

Fig. $4 E_{\mathrm{Rec}}$ distribution of the events arriving from the three off-zones with $\Lambda>-5.1$ : data (black crosses), $68 \%$ confidence area for the total background from simulation (grey area), $v_{\mathrm{atm}}^{\mathrm{sim}}$ (blue filled circles), $\mu_{\mathrm{atm}}^{\mathrm{sim}}$ (pink empty circles), expected signal from the Fermi bubbles according to (3)-(4) without neutrino energy cutoff (green dotted area) and with $50 \mathrm{TeV}$ energy cutoff (green dashed area). The expected signal was scaled by a factor of 3 to allow easy comparison with the total off-zone distribution

Table 1 Optimisation results for each cutoff of the neutrino energy spectrum

\begin{tabular}{lrrrr}
\hline$E_{\nu}^{\text {cutoff }}(\mathrm{TeV})$ & \multicolumn{1}{c}{$\infty$} & \multicolumn{1}{c}{500} & \multicolumn{1}{l}{100} & \multicolumn{1}{c}{50} \\
\hline$\Lambda^{\text {cut }}$ & -5.16 & -5.14 & $-5.14^{*}$ & -5.14 \\
$\log _{10}\left(E_{\mathrm{Rec}}^{\text {cut }}[\mathrm{GeV}]\right)$ & 4.57 & 4.27 & $4.03^{*}$ & 3.87 \\
$\bar{A}_{90 \%}$ & 2.67 & 4.47 & 8.44 & 12.43 \\
$\bar{A}_{90 \%}^{100}(100 \mathrm{TeV}$ cuts $)$ & 3.07 & 4.68 & 8.44 & 12.75
\end{tabular}

Average upper limits on the flux coefficient $\bar{A}_{90 \%}$ are presented in units of $10^{-7} \mathrm{GeV} \mathrm{cm}^{-2} \mathrm{~s}^{-1} \mathrm{sr}^{-1}$. Numbers with a star indicate the cut used for the $\bar{A}_{90 \%}^{100}$ calculation presented in the last row of the table

$\bar{A}_{90 \%}=A_{\text {theory }} \frac{\bar{s}_{90 \%}(b)}{s}$.

Table 1 reports the optimal cuts ( $\left.\Lambda^{\text {cut }}, E_{\mathrm{Rec}}^{\text {cut }}\right)$ obtained for the four chosen cutoff energies $(\infty, 500,100,50 \mathrm{TeV})$ of the neutrino source spectrum and the corresponding value of the average upper limit on the flux coefficient $\bar{A}_{90} \%$. Additionally, the optimal cuts for $E_{v}^{\text {cutoff }}=100 \mathrm{TeV}$ are applied for the other neutrino-energy cutoffs and the values $\bar{A}_{90 \%}^{100}$ are reported for comparison. As the obtained values $\bar{A}_{90 \%}$ and $\bar{A}_{90 \%}^{100}$ for each cutoff are similar, the $100 \mathrm{TeV}$ cuts are chosen for the final event selection.

At energies above $100 \mathrm{TeV}$ the semi-leptonic decay of short-lived charmed particles might become a major source of atmospheric neutrino background. The uncertainty in the flux from this contribution is large [23-25]. Due to the comparison of on and off zones (Sect. 5) and the final cut $\sim 10 \mathrm{TeV}$ (Table 1) the flux from charmed particle decays will not have 


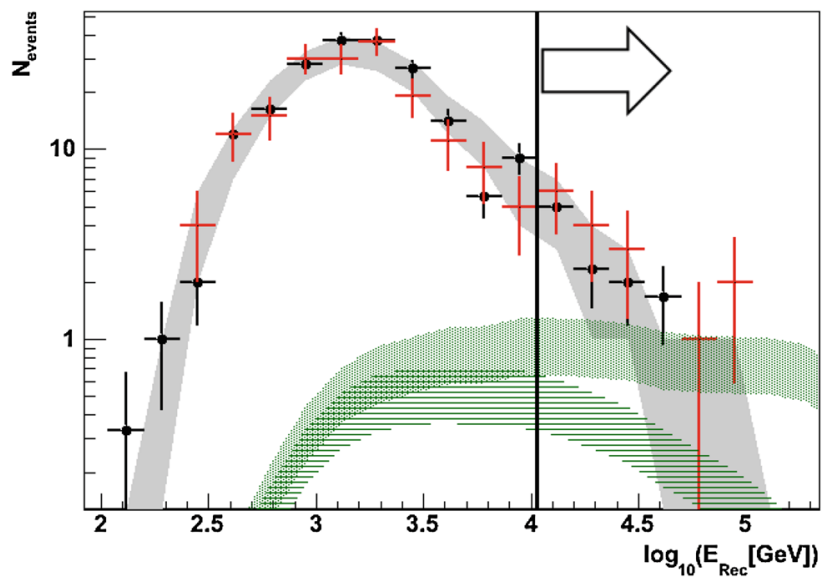

Fig. 5 Distribution of the reconstructed energy of the events after the final cut on $\Lambda$ : events in on-zone (red crosses), average over off-zones (black circles), $68 \%$ confidence area given by the total background simulation ( grey area), expected signal from the Fermi bubbles without neutrino-energy cutoff (green dotted area) and $50 \mathrm{TeV}$ cutoff (green dashed area). The chosen $E_{\mathrm{Rec}}^{\mathrm{cut}}$ is represented by the black line with an arrow

a significant impact on the analysis nor alter the final result on upper limits.

\section{Results}

The final event selection $\Lambda>-5.14, \log _{10}\left(E_{\mathrm{Rec}}[\mathrm{GeV}]\right)>$ 4.03 is applied to the unblinded data. In the three off-zones 9,12 and 12 events are observed. In the Fermi bubble regions $N_{\text {obs }}=16$ events are measured. This corresponds to $1.2 \sigma$ excess calculated using the method by $\mathrm{Li}$ and $\mathrm{Ma}$ [26].

The distribution of the energy estimator for both the onzone and the average of the off-zones is presented in Fig. 5. A small excess of high-energy events in the on-zone is seen with respect to both the average from the off-zones and the atmospheric neutrino simulation.

An upper limit on the number of signal events is calculated using a Bayesian approach at $90 \%$ coverage using the probability distribution with two Poisson distributions for the measurements in the on-zone and in the three off-zones. In order to account for systematic uncertainties in the simulation of the signal, a dedicated study has been performed in which the assumed absorption length in seawater is varied by $\pm 10 \%$ and the assumed optical module efficiency is varied by $\pm 10 \%$. For each variation the number of events is calculated for each cutoff and compared with the number of signal events $s$ obtained using the standard simulation. The differences are calculated and summed in quadrature to obtain $\sigma_{\text {syst }}$. A Gaussian distribution of the efficiency coefficient for the signal with mean $s$ and standard deviation $\sigma_{\text {syst }}$ is convoluted to the probability distribution. The maximum of the probability distribution is found for every
Table $290 \%$ confidence level upper limits on the neutrino flux coefficient $A_{90 \%}$ for the Fermi bubbles presented in units of $10^{-7} \mathrm{GeV} \mathrm{cm}^{-2} \mathrm{~s}^{-1} \mathrm{sr}^{-1}$

\begin{tabular}{lcccc}
\hline$E_{v}^{\text {cutoff }}(\mathrm{TeV})$ & $\infty$ & 500 & 100 & 50 \\
\hline Number of signal events in simulation $s$ & 2.9 & 1.9 & 1.1 & 0.7 \\
Uncertainty on the efficiency $\sigma_{\text {syst }}, \%$ & 14 & 19 & 24 & 27 \\
$A_{90 \%}$ & 5.4 & 8.7 & 17.0 & 25.9 \\
\hline
\end{tabular}

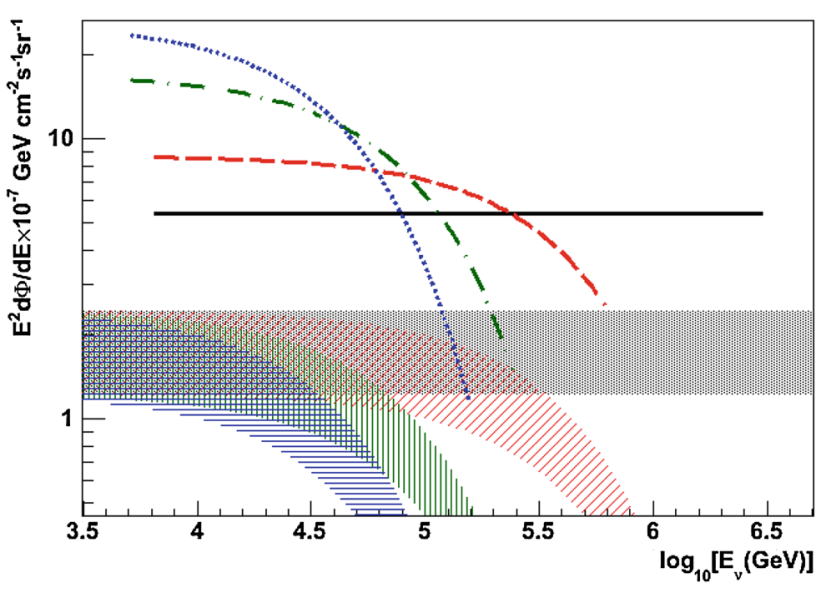

Fig. 6 Upper limits on the neutrino flux from the Fermi bubbles for different cutoffs: no cutoff (black solid), $500 \mathrm{TeV}$ (red dashed), $100 \mathrm{TeV}$ (green dot-dashed), $50 \mathrm{TeV}$ (blue dotted) together with the theoretical predictions for the case of a purely hadronic model (the same colours, areas filled with dots, inclined lines, vertical lines and horizontal lines respectively). The limits are drawn for the energy range where $90 \%$ of the signal is expected

neutrino flux coefficient $A$ and the obtained profile likelihood is used together with the flat prior for $A$ to calculate the postprobability. The upper and lower limits for $A$ are extracted from the post-probability to have $90 \%$ coverage.

The results are summarised in Table 2. A graphical representation of the upper limits on a possible neutrino flux together with the predicted flux is shown in Fig. 6. The obtained upper limits are above the expectations from the considered models. The modified Feldman and Cousins approach with the included uncertainties gives comparable results [27].

\section{Conclusions}

High-energy neutrino emission from the region of the Fermi bubbles has been searched for using data from the ANTARES detector. An analysis of the 2008-2011 ANTARES data yielded a $1.2 \sigma$ excess of events in the Fermi bubble regions, compatible with the no-signal hypothesis. For the optimistic case of no energy cutoff in the flux, the upper limit is within a factor of three of a prediction from the purely hadronic model based on the measured gamma-ray flux. The sensi- 
tivity will improve as more data is accumulated (more than $65 \%$ gain in the sensitivity is expected once 2012-2016 data is added to the analysis). The next generation KM3NeT neutrino telescope will provide more than an order of magnitude improvement in sensitivity [28-30].

Acknowledgments The authors acknowledge the financial support of the funding agencies: Centre National de la Recherche Scientifique (CNRS), Commissariat á l'Énergie Atomique et aux Énergies Alternatives (CEA), Agence National de la Recherche (ANR), Commission Européenne (FEDER fund and Marie Curie Program), Région Alsace (contrat CPER), Région Provence-Alpes-Côte d'Azur, Département du Var and Ville de La Seyne-sur-Mer, France; Bundesministerium für Bildung und Forschung (BMBF), Germany; Istituto Nazionale di Fisica Nucleare (INFN), Italy; Ministerio de Ciencia e Innovación (MICINN), Prometeo of Generalitat Valenciana and MultiDark, Spain; Agence de l'Oriental, Morocco; Stichting voor Fundamenteel Onderzoek der Materie (FOM), Nederlandse organisatie voor Wetenschappelijk Onderzoek (NWO), The Netherlands; National Authority for Scientific Research (ANCS-UEFISCDI), Romania; Council of the President of the Russian Federation for young scientists and leading scientific schools supporting grants, Russia. Technical support of Ifremer, AIM and Foselev Marine for the sea operation and the CC-IN2P3 for the computing facilities is acknowledged.

Open Access This article is distributed under the terms of the Creative Commons Attribution License which permits any use, distribution, and reproduction in any medium, provided the original author(s) and the source are credited.

Funded by $\mathrm{SCOAP}^{3}$ / License Version CC BY 4.0.

\section{References}

1. M. Su, T. Slatyer, D. Finkbeiner, Giant gamma-ray bubbles from Fermi-LAT: AGN activity or bipolar galactic wind? Astrophys. J. 724, 1044 (2010)

2. G. Dobler, A last look at the microwave haze/bubbles with WMAP. Astrophys. J. 750, 17 (2012)

3. E. Carretti et al., Giant magnetized outflows from the centre of the Milky Way. Nature 493, 66 (2013)

4. S.L. Snowden et al., ROSAT survey diffuse X-ray background maps. II. Astrophys. J. 485, 125 (1997)

5. R. Crocker, F. Aharonian, The Fermi bubbles: giant, multi-billionyear-old reservoirs of galactic center cosmic rays. Phys. Rev. Lett. 106, 101102 (2011)

6. B.C. Lacki, The Fermi bubbles as starburst wind termination shocks (2013) [arXiv:1304.6137]

7. S. Thoudam, Fermi bubble $\gamma$-rays as a result of diffusive injection of Galactic cosmic rays (2013) [arXiv:1304.6972]

8. D.O. Chernyshov et al., Particle acceleration and the origin of gamma-ray emission from Fermi bubbles. ICRC2011 proceedings (2011) [arXiv:1109.2619]

9. P. Mertsch, S. Sarkar, Fermi gamma-ray 'bubbles' from stochastic acceleration of electrons. Phys. Rev. Lett. 107, 091101 (2011)
10. G. Dobler, I. Cholis, N. Weiner, The Fermi gamma-ray haze from dark matter annihilations and anisotropic diffusion. Astrophys. J. 741, 25 (2011)

11. F.L. Villante, F. Vissani, How precisely neutrino emission from supernova remnants can be constrained by gamma ray observations? Phys. Rev. D 78, 103007 (2008)

12. ANTARES Collaboration, J.A. Aguilar et al., ANTARES: the first undersea neutrino telescope. Nuclear Inst. Methods Phys. Res. A 656, 11-38 (2011)

13. ANTARES Collaboration, S. Adrián-Martínez et al., The positioning system of the ANTARES neutrino telescope. JINST 7, T08002 (2012)

14. ANTARES Collaboration, J.A. Aguilar et al., Time calibration of the ANTARES neutrino telescope. Astropart. Phys. 34, 539 (2011)

15. ANTARES Collaboration, S. Adrián-Martínez et al., Search for cosmic neutrino point sources with four year data of the ANTARES telescope. Astrophys. J. 760, 53 (2012)

16. ANTARES Collaboration, J.A. Aguilar et al., A fast algorithm for muon track reconstruction and its application to the ANTARES neutrino telescope. Astropart. Phys. 34, 652-662 (2011)

17. J. Schnabel, Muon energy reconstruction in the ANTARES detector. Nuclear Inst. Methods Phys. Res. A 725, 106-109 (2013)

18. J. Brunner, ed. E. de Wolf, ANTARES simulation tools. VLVnT workshop (Amsterdam). http://www.vlvnt.nl/proceedings (2003)

19. Bartol Group, V. Argawal et al., Atmospheric neutrino flux above 1 GeV. Phys. Rev. D 53, 1314-1323 (1996)

20. D. Palioselitis, Measurement of the atmospheric neutrino energy spectru, $\mathrm{PhD}$ thesis work. http://antares.in2p3.fr/Publications/ thesis/2012/PhDThesis_Palioselitis (2012)

21. G.J. Feldman, R.D. Cousins, Unified approach to the classical statistical analysis of small signals. Phys. Rev. D 57, 3873-3889 (1998)

22. G.C. Hill, K. Rawlins, Unbiased cut selection for optimal upper limits in neutrino detectors: the model rejection potential technique. Astropart. Phys. 19, 393-402 (2003)

23. C.G.S. Costa, The prompt lepton cookbook. Astropart. Phys. 16, $193(2001)$

24. A. Martin, M. Ryskin, A. Stasto, Prompt neutrinos from atmospheric $c \bar{c}$ and $b \bar{b}$ production and the gluon at very small $x$. Acta Phys. Pol. B 34, 3273 (2003)

25. R. Enberg, M. Reno, I. Sarcevic, Prompt neutrino fluxes from atmospheric charm. Phys. Rev. D 78, 043005 (2008)

26. T. Li, Y. Ma, Analysis methods for results in gamma-ray astronomy. Astrophys. J. 272, 317-323 (1983)

27. J. Conrad et al., Including systematic uncertainties in confidence interval construction for Poisson statistics. Phys. Rev. D. 67, $012002(2003)$

28. KM3NeT Collaboration, S. Adrián-Martínez et al., Detection potential of the KM3NeT detector for high-energy neutrinos from the Fermi bubbles. Astropart. Phys. 42, 7-14 (2013)

29. C. Lunardini, S. Razzaque, High energy neutrinos from the Fermi bubbles. Phys. Rev. Lett. 108, 221102 (2012)

30. I. Cholis, Searching for the high energy neutrino counterpart of the Fermi bubbles signal or from dark matter annihilation (2012) [arXiv:1206.1607] 